\title{
Adult Primary Tuberculosis of Lateral End of Clavicle - A Case of Missed Diagnosis
}

\author{
Sathish Muthu ${ }^{1}$, Girinivasan Chellamuthu ${ }^{1}$
}

Learning Point of the Article:

Primary tuberculosis of the clavicle is a rarity that mimics other diseases like a tumor; hence, clinicians should be aware of a wide range of presentations to diagnose such an entity.

\section{Abstract}

Introduction: Of the largest series on osteoarticular tuberculosis (TB) by Tuli et al. involving 1074 cases, only seven involved clavicle. It is also noted that the lateral end of the clavicle has been less frequently involved by tubercular disease than the medial end. We present a case of primary tubercular involvement of the lateral end of the clavicle and discuss its management by an innovative modification of the Weaver-Dunn procedure.

Case Report: Twenty-five-year-old working women came with complaints of swelling in her left shoulder for the past 2 months. On examination, swelling with restricted shoulder movements was noted. Radiological evaluation revealed an osteolytic lesion with a pathological fracture in the lateral end of the clavicle. With a provisional diagnosis of giant cell tumor, we proceeded with fine-needle aspiration cytology which was inconclusive. Blood parameters were within normal limits. We proceeded with the excision biopsy of the lateral end of the clavicle and stabilization with a modified Weaver-Dunn procedure involving the reconstruction of the coracoclavicular ligament and augmentation with an Endobutton loop. Biopsy reported the lesion to be of tuberculous etiology. The patient made a good recovery without any complications.

Conclusion: Primary TB of the clavicle is a rarity that mimics other diseases like a tumor; hence, clinicians should be aware of a wide range of presentations to diagnose such an entity. Although antitubercular treatment remains the mainstay of treatment, surgical resection is justified in cases of uncertain diagnosis or disease not responding to conservative lines of management and in cases that need the removal of the sequestrum. Keywords: Primary tuberculosis, clavicle, modified Weaver-Dunn procedure, osteoarticular tuberculosis, pathological fracture.

\section{Introduction}

Osteoarticular involvement of tuberculosis (TB) contributes only $2-5 \%$ of all the tubercular lesions in the body [1]. The axial skeleton is predominantly involved, making tubercular lesions of the appendicular skeleton a rarity [2]. Moreover, such appendicular involvements are mostly reported in children, making adult presentations even rarer [3]. Although TB is known to be a mimicker of all diseases, the absence of pulmonary lesions and classical symptomatology makes their primary diagnosis even more difficult [4].

Of the largest series on osteoarticular TB by Tuli et al. involving 1074 cases, only seven involved clavicle [5]. It is also noted that the lateral end of the clavicle has been less frequently involved by tubercular disease than the medial end [6]. In this background, we present a case of primary tubercular involvement of the lateral end of the clavicle and discuss its management by an innovative modification of the WeaverDunn procedure [7].

\section{Case Report}

Twenty-five-year-old working women came with complaints of swelling in the left shoulder for the past 2 months, associated with pain for the past 1 month. She had difficulty using her shoulder. There was no significant history of trauma. No history

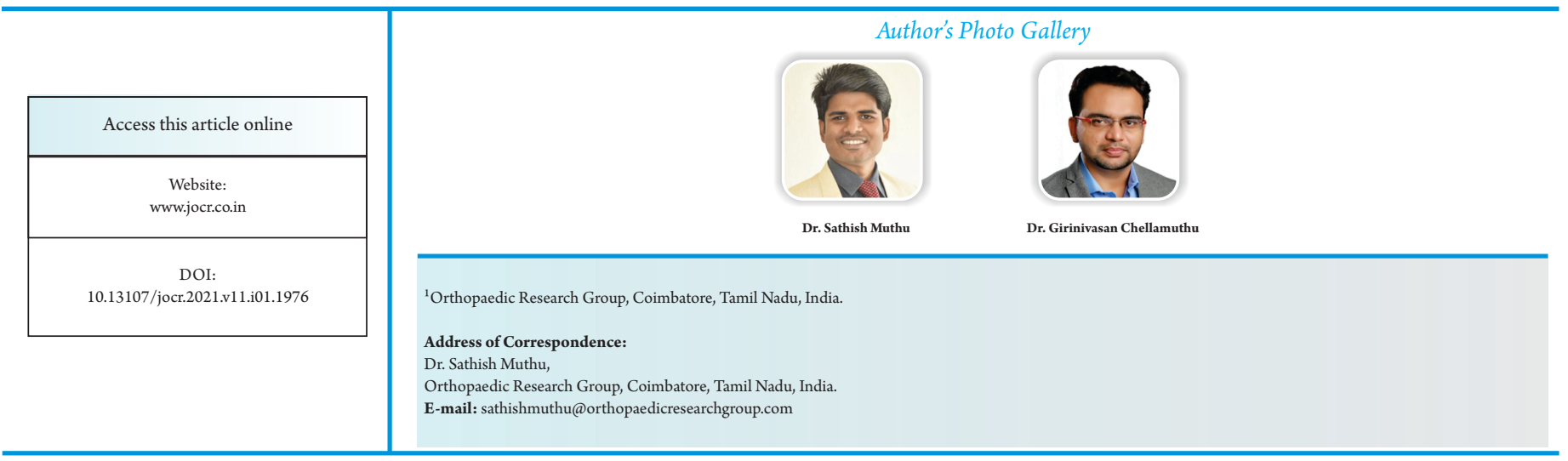

Journal of Orthopaedic Case Reports | pISSN 2250-0685 | eISSN 2321-3817 | Available on www.jocr.co.in | doi:10.13107/jocr.2021.v11.i01.1976 This is an Open Access article distributed under the terms of the Creative Commons Attribution Non-Commercial License (http://creativecommons.org/licenses/by-nc/3.0) which permits unrestricted non-commercial use, distribution, and reproduction in any medium, provided the original work is properly cited. 

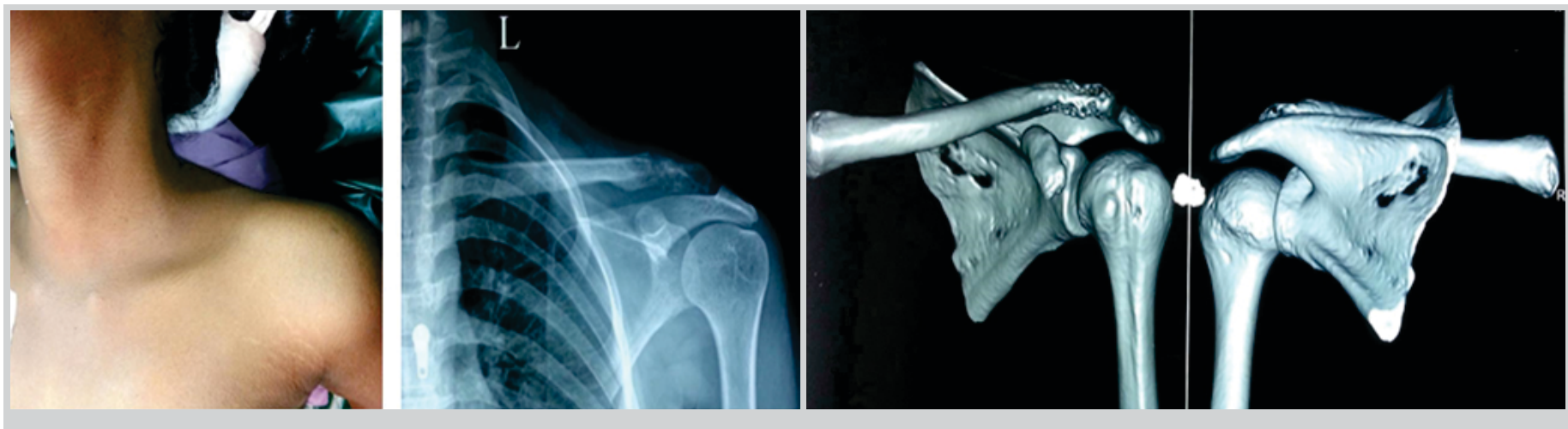

Figure 1: The clinical and radiological evaluation of the osteolytic lesion in the lateral end of the clavicle.

of constitutional symptoms suggestive of infection or tumor.

On examination, tender swelling of size $4 \times 3 \mathrm{~cm}$ was noted in the lateral end of the clavicle with restricted shoulder movements. The skin over the swelling was normal without any sinus or dilated veins, as shown in (Fig. 1).

\section{Investigations}

Radiological evaluation revealed an osteolytic lesion in the lateral end of the clavicle with pathological fracture, minimal periosteal reaction, and rarefaction without calcification or soft -tissue involvement, as shown in (Fig. 1). Magnetic resonance imaging (MRI) showed multiple septations within the lesion with relatively well-defined borders.

With a provisional diagnosis of giant cell tumor, we proceed to fine-needle aspiration cytology (FNAC) of the lesion. It showed a sparsely cellular smear with osteoclastic giant cells among dispersed stromal cells which were not diagnostic but suggestive of a giant cell tumor. All her blood investigations were within normal limits including white cell counts, erythrocyte sedimentation rate, and C-reactive protein estimates.

\section{Treatment}

Since the patient had a doubtful diagnosis with a pathological fracture and a painful range of movements, we planned for excision biopsy of the lesion by resection of the lateral end of the clavicle followed by a stabilization procedure for the lateral end of the clavicle. It was noted from the pre-operative radiograph
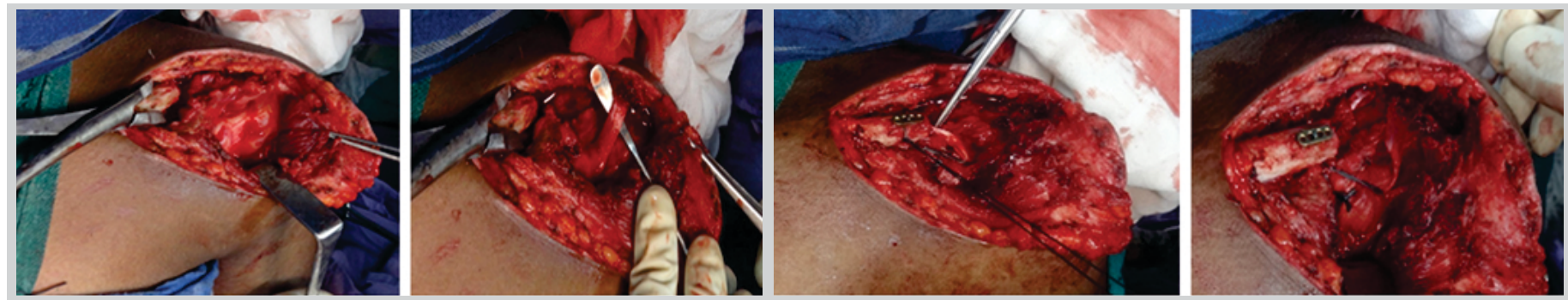

Figure 2: The exposure of the lesion, identification of the coracoacromial ligament (CAL) after excision of the lesion, reconstruction of the CAL from the CAL, augmentation of the reconstruction with coracoclavicular loop sutured over Endobutton on the clavicle.

Journal of Orthopaedic Case Reports | Volume 11 | Issue 1 | January 2021 | Page 87-90 that a stabilization procedure like a coracoclavicular screw would not be possible due to the extent of the tumor and hence an alternate surgical procedure like the Weaver-Dunn procedure [7] was contemplated.

Through Robert's subcutaneous approach for clavicle, the lesion is exposed and the lateral end of the clavicle with a clear margin was resected. The coracoacromial ligament was identified and used in the reconstruction of the coracoclavicular ligament, as shown in (Fig. 2). We modified the described Weaver-Dunn procedure by augmenting the reconstruction with Ethibond looped from the coracoid to be tunneled through the inferior surface of the clavicle to be tied on its superior surface with Endobutton to reduce the strain on the reconstructed ligament to aid in the healing process, as shown in (Fig. 2). Biopsy results diagnosed the lesion to be of tuberculous origin exhibiting a collection of lymphocytes, plasma cells, epithelioid cells, and multinucleated giant cells. Hence, the patient was started on anti-tuberculous treatment. Postoperatively, the patient was kept on an arm sling for 2 weeks and proceeded with graded mobilization exercises. On further evaluation with computed tomography chest for any occult primary in the lungs was uneventful making the case primary TB of clavicle.

\section{Outcome and follow-up}

The patient showed good recovery with an improved painless range of movements and resumed her routine activities by 6 weeks. She completed her anti-tuberculous treatment course 


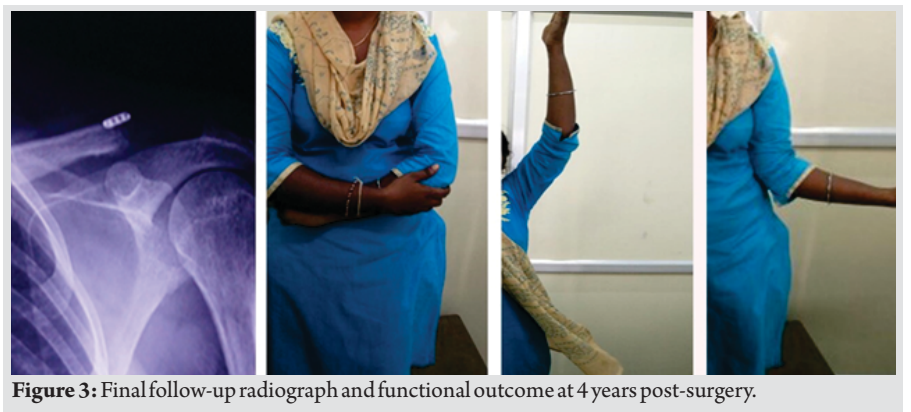

for 9 months without any complications. At 4-year follow-up, she had excellent clinical outcome scores of $46 / 60$ and 88/100 as measured with Oxford Shoulder Score [8] and Nottingham Clavicle Score [9], respectively, as shown in (Fig. 3).

\section{Discussion}

TB rarely involves flat bones of the chest and skull making clavicular involvement an exceptional phenomenon [10]. TB infection of the clavicle has a wide range of presentations from dull aching pain to swelling and draining sinus [11]. The diagnostic dilemma of this rare presentation is confounded by the fact that the location is more prone to neoplasms compared to infections [12].

There are two patterns of involvement: Proliferative type and destructive type [13]. Although plain radiographs may show features such as diffuse thickening, multiple cystic cavities, and honeycombing to differentiate them, due to the overlapping anatomical structures, MRI remains the investigation of choice which shows loss of normal hypodense cortices [14].

Extrapulmonary osteoarticular TB almost always has a primary focus elsewhere in the body and a definite attempt must be made to screen the pulmonary, gastrointestinal, and renal system for any evidence of the disease [15]. Despite our attempted screening for the identification of primary focus, we did not find any such lesions making our case primary TB of clavicle, which is rarer. For a definite diagnosis of TB, a representative sample either by FNAC or biopsy is needed to identify the pathogen or its disease process [16]. It is not always possible to isolate the bacteria from the lesion even on culture $[17,18]$. Hence, supportive evidence in biopsy samples drives us toward the diagnosis as in our case.

Various authors noted a considerable delay before a diagnosis could be made in such conditions which are attributed to the

\section{References}

1. Tuli SM. General principles of osteoarticular tuberculosis. Clin Orthop Relat Res 2002;398:11-9.

2. Parmar H, Shah J, Patkar D, Singrakhia M, Patankar T, Hutchinson C. Tuberculous arthritis of the appendicular skeleton: MR imaging appearances. Eur J Radiol slow insidious pathologic process $[19,20]$. Factors that contribute to such delays are mainly its rarity of presentation in such unusual sites with its ability to mimic other diseases combined with the lack of suspicion by the treating clinician, especially in non-endemic areas. With time, the lesion would present itself either as a discharging sinus or with the involvement of the adjacent joint which further adds to the complications such as superimposed infections and impaired join function on healing [21].

Surgical excision is justified in cases of uncertain diagnosis or disease not responding to conservative lines of management and in cases that need the removal of the sequestrum [13]. Since we noted an isolated expansile lytic lesion without any primary complex with supportive FNAC findings, we made a provisional diagnosis of giant cell tumor and proceeded for excision biopsy and reconstruction. The Weaver-Dunn procedure was described for the management of acute or chronic acromioclavicular joint injuries which includes the transfer of coracoacromial ligament to the distal end of the resected clavicle as described by Mumford [22]. We utilized it for the stabilization of the cut end of the clavicle with modification, as shown in (Fig. 2), which provided the necessary environment for the healing of the reconstructed ligaments to yield a good functional result.

Although there are very few case reports on similar presentations in literature $[3,11,23,24]$, this is case reports on the combined medical and surgical management of the destructive type of TB infection of the lateral end of the clavicle in an adult.

\section{Conclusion}

Primary TB of the clavicle is a rarity that mimics other diseases like a tumor; hence, clinicians should be aware of a wide range of presentations to diagnose such an entity. Although ATT remains the mainstay of treatment, surgical resection is justified in cases of uncertain diagnosis or disease not responding to conservative lines of management and in cases that need the removal of the sequestrum.

\section{Clinical Message}

Although rare, tuberculous etiology should be considered for the atypical presentation of any osseous pathology as illustrated in this case and managed accordingly.

3. Dugg P, Shivhare P, Mittal S, Singh H, Tiwari P, Sharma A. Clavicular osteomyelitis: A rare presentation of extra pulmonary tuberculosis. J Surg Case Rep 2013;2013:rjt030. 
4. Prapruttam D, Hedgire SS, Mani SE, Chandramohan A, Shyamkumar NK, Harisinghani M. Tuberculosis the great mimicker. Semin Ultrasound CT MR2014;35:195-214.

5. Tuli SM, Sinha GP. Skeletal tuberculosis "unusual lesions". Indian J Orthop 1969;3:5-19.

6. Meena UK, Saibaba B, Behera P, Meena RC. Sternoclavicular joint tuberculosis: A series of 9 cases. Indian J Tuberc 2017;64:221-4.

7. Weaver JK, Dunn HK. Treatment of acromioclavicular injuries, especially complete acromioclavicular separation. J Bone Joint Surg Am 1972;54:1187-94.

8. Younis F, Sultan J, Dix S, Hughes PJ. The range of the oxford shoulder score in the asymptomatic population: A marker for post-operative improvement. Ann R Coll Surg Engl 2011;93:629-33.

9. Charles ER, Kumar V, Blacknall J, Edwards K, Geoghegan JM, Manning PA, et al. A validation of the Nottingham clavicle score: A clavicle, acromioclavicular joint and sternoclavicular joint-specific patient-reported outcome measure.J Shoulder Elbow Surg 2017;26:1732-9.

10. Mishra SK, Nigam P. Tuberculosis of flat bones. Indian J Chest Dis Allied Sci 1984;26:174-6.

11. Basanagoudar PL, Gupta PN, Bahadur R, Dhillon MS. Tuberculosis of the clavicle presenting as an expansile lytic lesion: A case report. Acta Orthop Belg 2001;67:505-9.

12. Mikawa T, Miyoshi K, Fujita K, Hase R, Hosokawa N. The great imitator; clavicular tuberculosis mimics a metastatic neoplasm. Kansenshogaku Zasshi 2015;89:588-91.

13. Tuli SM. Tuberculosis of the Skeletal System. 3rd ed. New Delhi:Jaypee Brothers; 2004.p. 167-73.
14. Jain AK, Jena SK, Singh MP, Dhammi IK, Ramachandran VG, Dev G. Evaluation of clinico-radiological, bacteriological, serological, molecular and histological diagnosis of osteoarticular tuberculosis. Indian J Orthop 2008;42:173-7.

15. Agathangelidis F, Boutsiadis A, Fouka E, Karataglis D. Concomitant acromioclavicular and miliary tuberculosis. BMJ Case Rep 2013;2013:bcr2013010026.

16. Reisis N, Dendrinos G, Fragiadakis E, Trouli H, Hartofylakides G. Value of tissue biopsy in bone and joint tuberculosis. Acta Orthop Belg 1989;55:12-6.

17. Vardhan V, Yanamandra U. Diagnosis of osteoarticular tuberculosis. Indian J Rheumatol 2011;6:87-94.

18. Hunt DD. Problems in diagnosing osteoarticular tuberculosis.JAMA 1964;190:95-8.

19. Rajasekaran S. Tuberculosis of bones, joints, and spine: Evidence-based management guide. Indian J Orthop 2017;51:721.

20. Prakash J, Sareen A, Arora P, Chopra RK. Sternoclavicular tuberculosis: An atypical imitator of refractory shoulder pain. Int Orthop 2020;44:693-8.

21. Sequeira W, Co H, Block JA. Osteoarticular tuberculosis: Current diagnosis and treatment. Am J Ther 2000;7:393-8.

22. Mumford E. Acromioclavicular dislocation. A new operative treatment.J Bone Joint Surg 1941;23:799-802.

23. Aggarwal A, Dhammi I, Singh A, Kumar S, Goyal M. Tubercular osteomyelitis of the clavicle: A report of four cases.J Orthop Surg (Hong Kong) 2009; 17:123-6.

24. Prakash J, Aggarwal S, Mehtani A. Primary Tuberculosis of the Clavicle. Orthopedics 2014;37:e879-84.

Conflict of Interest: Nil

Source of Support: Nil

Consent: The authors confirm that informed consent was obtained from the patient for publication of this case report

\section{How to Cite this Article}

Muthu S, Chellamuthu G. Adult primary tuberculosis of lateral end of clavicle - A case of missed diagnosis. Journal of Orthopaedic Case Reports 2021 January; 11(1): 87-90. 